\title{
The effect of calcium sulfate/calcium phosphate composite for the treatment of chronic osteomyelitis compared with calcium sulfate
}

\author{
Zihou Zhao" ${ }^{1 \#}$, Guoliang Wang ${ }^{1 \#}$, Yong Zhang ${ }^{1 \#}$, Wen Luo $^{2}$, Shiyu Liu ${ }^{3}$, Yunyan Liu ${ }^{1}$, Yong Zhou ${ }^{1}$, \\ Yunfei Zhang ${ }^{1}$
}

${ }^{1}$ Department of Orthopaedics, Second Affiliated Hospital, Air Force Medical University, Xi'an, China; ${ }^{2}$ Department of Ultrasound, Xijing Hospital, Air Force Medical University, Xi'an, China; ' Institute of Oral Tissue Engineering, Air Force Medical University, Xi'an, China

Contributions: (I) Conception and design: Z Zhao, Y Zhang, S Liu; (II) Administrative support: Y Zhang, Y Zhou; (III) Provision of study materials or patients: None; (IV) Collection and assembly of data: G Wang, Y Liu; (V) Data analysis and interpretation: All authors; (VI) Manuscript writing: All authors; (VII) Final approval of manuscript: All authors.

\#These authors contributed equally to this work.

Correspondence to: Yunfei Zhang, PhD; Yong Zhou, PhD. Department of Orthopaedics, Second Affiliated Hospital, Air Force Medical University, Xi’an 710038, China. Email: tdbone@163.com; gukezy@fmmu.edu.cn.

Background: Treatment of chronic osteomyelitis often requires surgical debridement in combination with bone defect reconstruction and antibiotics administration. We aimed at investigating and evaluating the effect of antibiotic-loaded absorbable calcium sulfate/calcium phosphate (CS/CP) composite as bone substitute in the treatment of chronic osteomyelitis compared with CS.

Methods: A retrospective study of 31 consecutive patients with chronic osteomyelitis from one medical center was conducted. The treatment involved thorough debridement, antibiotic loaded bone substitutes filling (group A: CS/CP, 21 patients, group B: CS, 10 patients), laboratory and radiographic examination, and culture-specific systemic antibiotic treatment guided by a multidisciplinary team. New bone formation property and resorption kinetics were analyzed through X-ray and CT scan qualitatively and quantitatively. Anti-infection effect was mainly analyzed by postoperative laboratory examination and healing of wound.

Results: The average follow-up in each group was 61.3 and 86.7 weeks, respectively. In group A (CS/ $\mathrm{CP})$, no patient had recurrent infection at 17 months after surgery, 1 case had delayed wound healing and healed after dressing change. In group B (CS), 2 patients had recurrent infection at 18 weeks after surgery, and were managed after further surgical treatment, 3 cases had delayed wound healing and healed after dressing change. The infection in the two groups was mainly caused by staphylococcus aureus. The average percentage of new bone formation was $20.5 \%, 43.7 \%, 75.2 \%$ at 1,3 , and 6 months in group A and $15.4 \%$, $32.2 \%, 49.7 \%$ at 1,3 , and 6 months in group $\mathrm{B}$ after operation $(\mathrm{P}=0.001$ at 1 month, $\mathrm{P}=0.025$ at 3 months, $\mathrm{P}=0.000$ at 6 months). The average percentage of resorption was $23.7 \%, 56.4 \%, 81.2 \%$ at 1,3 , and 6 months in group $\mathrm{A}$ and $47.1 \%, 96.2 \%, 100 \%$ at 1,3 , and 6 months in group B after operation ( $\mathrm{P}=0.000$ at 1 month, $\mathrm{P}=0.000$ at 3 months, $\mathrm{P}=0.000$ at 6 months). There was difference in infection recurrence $(\mathrm{P}=0.034)$.

Conclusions: Our preliminary results proved that compared with CS, this novel antibiotic-impregnated CS/CP composite acted as superior scaffold for bone formation with a lower rate of infection recurrence, when choosing bone substitutes in the treatment of chronic osteomyelitis.

Keywords: Calcium phosphate (CP); calcium sulfate (CS); osteomyelitis

Submitted Jan 11, 2020. Accepted for publication Feb 10, 2020.

doi: 10.21037/apm.2020.03.23

View this article at: http://dx.doi.org/10.21037/apm.2020.03.23 


\section{Introduction}

Chronic osteomyelitis has always been a troublesome problem in the field of orthopedic surgery due to long period of treatments and poor prognosis. The etiology of chronic osteomyelitis can be summarized as acute hematogenous osteomyelitis, post traumatic infection (especially after internal fixation), and soft tissue infection $(1,2)$. Thorough debridement acts as the key process during surgical treatment, which inevitably leads to enlarged bone defects (3). Staphylococcus aureus is one of the most common bacteria related with osteomyelitis (4). The treatment of osteomyelitis requires not only reconstruction of bone defect but also effective and long-term control of infection.

Traditionally, autologous bone graft has been the gold standard for all grafting procedures due to osteoconduction, osteoinduction and osteogenic properties (5). However, it has apparent disadvantages such as limited supply and donor site complications (6). Artificial synthetic bone substitutes like calcium sulfate $\left(\mathrm{CaSO}_{4}, \mathrm{CS}\right)$ or calcium phosphate $\left(\mathrm{CaPO}_{4}, \mathrm{CP}\right)$ are reasonable alternatives, because they are biodegradable and osteoconductive. In addition, they can be also used as the vector for antibiotic release which adds the effect of anti-infection (7).

CS as biodegradable ceramic has been used as bone graft material since 1892 (8). It has fast absorption and good histocompatibility properties. However, the mechanical strength of CS is only comparable to that of cancellous bone, and is hydrolyzed very quickly in bone, lasting for about 6-12 weeks. This means that it degrades too fast with low mechanical properties, and cannot provide enough structural support for bone ingrowth (9-11). CP has the characteristic of biocompatible and bioresorbable, meanwhile, it is similar to the inorganic bone structure of human body with porous features which improves osteoconductive ability (12-15). There are few complications (infection or nonunion) in the implantation of CP (14). However, its degradation rate is very slow, and studies showed that absorption of $\mathrm{CP}$ were completed in 20 weeks or even longer $(15,16)$. To overcome these shortcomings, $\mathrm{CaSO}_{4}-\mathrm{CaPO}_{4}$ composite graft with high compressive strength and intermediate bone absorption kinetics has become available $(17,18)$. And in recent studies, Urban RM showed the compressive stress was greater in defects treated with CS/CP than CS and normal bone, Yang reported that CS/CP acquired excellent mechanical properties up to $35 \mathrm{MPa}$ in compressive strength $(9,19)$.
This new hybrid material consists of matrix of $\mathrm{CaSO}_{4}$ and dicalcium phosphate (DCP) with beta-tricalcium phosphate ( $\beta$-TCP) granules (19). Thus, the composite has the triphasic degradation profile. Firstly, with the rapid dissolution and absorption of CS, leaving porous space for the formation of new born trabeculae and vascular growth to the remaining CP scaffold. Then, DCP degrades by osteoclastic resorption with an intermediated resorption profile. Finally, $\beta$-TCP undergoes osteoclastic degradation and remained longest (20-22).

Theoretically, due to the superior resorption profile, $\mathrm{CS} / \mathrm{CP}$ should have superior bone restoration property. Preclinical studies have showed that this hybrid composition could enhance the new bone formation compared with autograft, TCP alone or CS alone (19,21-25). Some recent clinical studies also confirmed the excellent radiological results in the treatment of femoral head avascular necrosis or benign bone lesions reconstruction (26-29).

It has also been proved that antibiotic-loaded CS is effective when treating chronic osteomyelitis due to antibiotic elution. Animal research proved that CS/CP composite can be used as an excellent delivery system for antibiotics (30). However, whether antibiotic-loaded CS/ $\mathrm{CP}$ can present superior property in the clinical treatment of chronic osteomyelitis is unknown. For the first time, we present the clinical report of this novel antibioticimpregnated CS/CP composition in the treatment of chronic osteomyelitis and antibiotic-impregnated CS was used as comparation.

The aim of our study was to investigate and evaluate the effect of CS/CP composite as bone substitute in the treatment of chronic osteomyelitis compared with CS. We specifically focus on (I) new bone formation rate, (II) graft resorption rate, and (III) anti-infection effect: complication and recurrence rate.

\section{Methods}

\section{Grouping}

A total of 31 patients with chronic osteomyelitis were included in this retrospective study during 2016-2018 in one medical center. Twenty-one patients (14 males 67\%, and 7 females), with an average age of $43(43.05 \pm 17.98)$ were enrolled as group A and were treated with vancomycin-impregnated CS/CP composites (PRODENSE ${ }^{\circledR}$ Wright Medical Group, Memphis, TN, USA), who had a mean follow-up of 61 weeks. Of these, 
Table 1 Preoperative data of the two groups $(\bar{x}+s)$

\begin{tabular}{lcc}
\hline & Group A (CS/CP) & Group B (CS) \\
\hline Total number of patients & 21 & 10 \\
Male/female & $14 / 7$ & $10 / 0$ \\
Age (years), mean \pm SD & $43.05 \pm 17.98$ & 0.066 \\
Pathogenesis (haematogenous/post-traumatic) & $8 / 13$ & 0.471 \\
Site (humerus/femur/tibia/ischium/forefoot and calcaneum) & $1 / 2 / 8 / 2 / 8$ & 1.000 \\
Pre-op ESR (mm/h), mean \pm SD & $12.51 \pm 13.09$ & $0.5 / 5 / 0 / 0$ \\
Pre-op serum hs-CRP (mg/L), mean \pm SD & $15.31 \pm 29.28$ & $17.10 \pm 21.74$ \\
Pre-op WBC $\left(\times 10^{9}\right)$, mean \pm SD & $6.31 \pm 3.65$ & $5.76 \pm 9.03$ \\
\hline
\end{tabular}

*, $\mathrm{P}<0.05$. ESR, erythrocyte sedimentation rate; hs-CRP, high-sensitivity C-reactive protein; WBC, white blood cell count; CS, calcium sulfate; $\mathrm{CP}$, calcium phosphate.

13 patients were post-traumatic, 8 patients had hematogenous osteomyelitis that led to infected bone defects. The distribution of bone defects was as follows: 1 patient in humerus, 2 patients in femur, 8 patients in tibia, 2 patients in ischium, 8 patients in calcaneus and forefoot. In contrast, 10 patients (10 males $100 \%$, and 0 female), with an average age of $48(48.2 \pm 19.22)$ were enrolled as group B and were treated with vancomycin-impregnated CS (OSTEOSET ${ }^{\circledR}$ Wright Medical Group, Memphis, TN, USA), who had a mean follow-up of 87 weeks. Of these, 6 patients were posttraumatic, 4 patients had hematogenous osteomyelitis that led to bone defects. The distribution of the bone defects was 5 patients in femur, and 5 patients in tibia (Table 1).

\section{Inclusion criteria and exclusion criteria}

Inclusion criteria: patients with definite history of trauma or hematogenous osteomyelitis and clinically diagnosed as chronic osteomyelitis by orthopedic surgeons, and who agreed to be hospitalized for surgical treatment. The diagnosis of the chronic osteomyelitis was confirmed by imaging and laboratory tests after admission. Meanwhile, the defect was less than $7 \mathrm{~cm}$ and there were no fractures of the same limb, good soft tissue condition was required. No serious cardiovascular system disease, hypohepatia, renal dysfunction or connective tissue disease were observed. Exclusion criteria was as follows: acute osteomyelitis with systemic symptoms, low immune function or acquired immune deficiency syndrome, diabetes or systemic malnutrition, allergic to CS or CP, severe cardiovascular diseases, liver or kidney dysfunction.

We obtained the informed consent of all subjects who were to participate in our study, and our research received permissions from the ethics committee of our institution, and was performed with their guidance and help (Assigned No. TDLL-201709-19).

\section{Surgical management}

The principles of chronic osteomyelitis treatment in both groups were as following: firstly, debridement, the necrotic infected bone and soft tissues as well as partial normal tissue were excised to make sure of radical debridement as much as possible. Then we measured the length and width of the bone defect area to estimate the dose of bone substitute required (Figure 1A,B). The bone defect cavity was irrigated using sterile saline for 3 times, followed by diluted iodophor saline soaking for $5 \mathrm{~min}$, and then sterile tablecloths and gloves were changed.

Secondly, bone substitute was implanted. Then the vancomycin-impregnated CS/CP composite was prepared. The outer packaging was opened using the standard aseptic "no touch" technique to avoid contact with the contents. The CS/CP powder was emptied into the sterile mixing bowl. The pre-measured sterile mixing solution was added and allowed to stand (soak-out) for one minute (Figure 1C). The mixture was thoroughly mixed until a paste was formed (approximately 30 seconds). After that, it was introduced into the syringe quickly, and then the composite starts to set 2-3 minutes after mixing. Vancomycin $300 \mathrm{mg} / \mathrm{cc}$ was mixed and stirred under negative pressure as previously described (Figure 1D) (31). In group A, the composite was filled into the bone defect area and then dried for 20-30 min (Figure 1E). The deep fascia and subcutaneous tissue were 

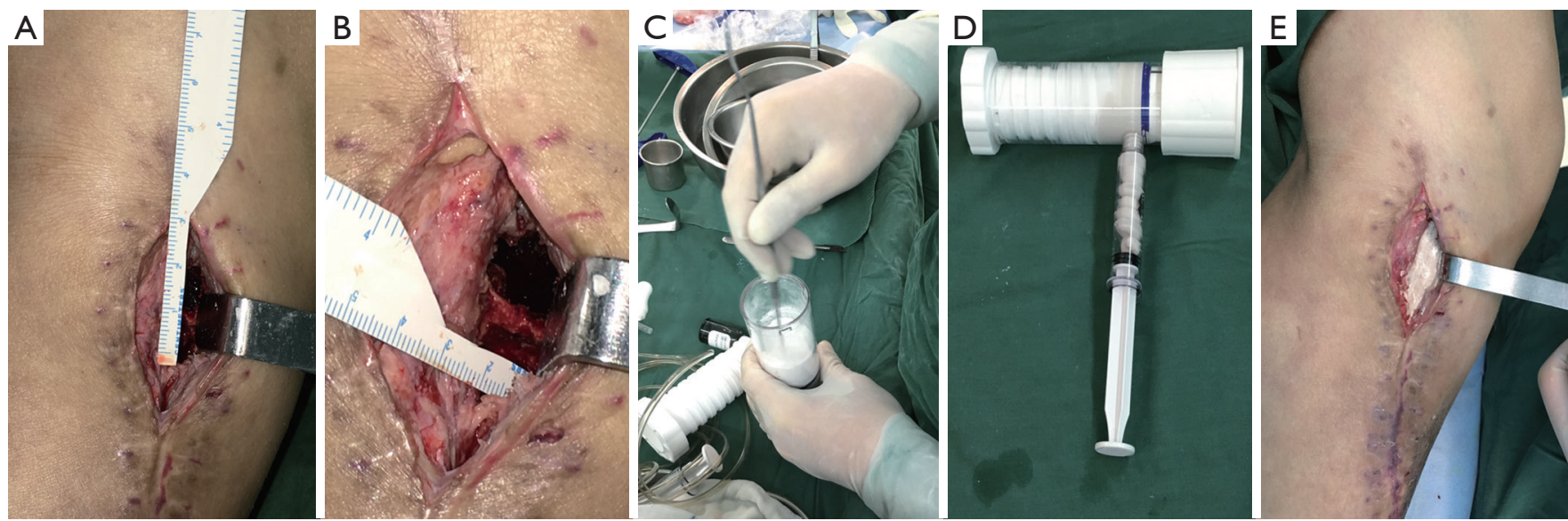

Figure 1 Patient with chronic osteomyelitis of tibia treated by CS/CP composite. (A,B) Debridement of infected bone and soft tissue was done, and the length and width of the bone window were measured. (C) Make the vancomycin-impregnated CS/CP composite intraoperatively by mixing CS/CP powder with solution. (D) The composite starts to set after mixing for 2-3 minutes. (E) The CS/CP composite was fully filled into the bone defect area without any cavity. CS, calcium sulfate; CP, calcium phosphate.

sutured layer by layer. The negative pressure suction drains were left and the dead cavity was completely eliminated. The incision was closed with a compression bandage. Group B patients were treated similarly, but the defect was filled with vancomycin-impregnated CS.

Thirdly, Antibiotic administration. Except for antibiotics local delivery, systemic intravenous or oral antibiotics were also used depend on results of bacterial culture and drug sensitivity analysis. The period usually lasted for 4-6 weeks according to the laboratory examination.

\section{New bone formation and substitute absorption analysis}

X ray, CT scan and Image Pro Plus 6.0 (Media Cybernetics, US) was used to measure and evaluate the rate of new bone formation and resorption of substitutes (new bone formation percentage $=$ new bone formation area/the whole defect area $\times 100 \%$, percentage of substitutes resorption $=1-$ remained bone substitutes area/the whole defect area $\times 100 \%)$. Three fields were randomly selected on the same CT scan and 3 values were obtained according to the above formula, and the mean value was taken and calculated by 2 different observers.

\section{Anti-infection and recurrence analysis}

Laboratory examinations including: erythrocyte sedimentation rate (ESR), WBC count, C-reactive protein (CRP), and clinical manifestation including skin color, temperature and wound drainage were used to analyze the antiinfection effect and infection recurrence. The results of microbial culture and drug sensitivity were identified. All microbiological results were assessed by senior physicians who were not involved in this study. The patients received follow-up at the time point of 1, 3, 6, 9 months and 1.5 years after operation.

\section{Statistical analysis}

We used Excel software to collect and record data after obtaining all relevant data, IBM SPSS statistics software (Version 23. 0) and Statistical Analysis System (SAS Version 9.4) were used for data analysis. Chi-square for categorical variables and $t$-test for continuous variables were performed in this study. Fisher's exact test was performed when the count was expected to be less than 5 . Pearson correlation coefficients were calculated for substitute filling dose and new bone formation and bone absorption. $\mathrm{P}$ values $<0.05$ were considered to be significant.

\section{Results}

\section{Preoperative and postoperative data analysis}

In group A, the mean dose of CS/CP composite and the antibiotic load were $3.95 \mathrm{cc}$ mixed with vancomycin $(300 \mathrm{mg} / \mathrm{cc})$. The average length of defect was $5.28 \pm 0.98 \mathrm{~cm}$, 
Table 2 Postoperative data of the two groups $(\bar{x}+s)$

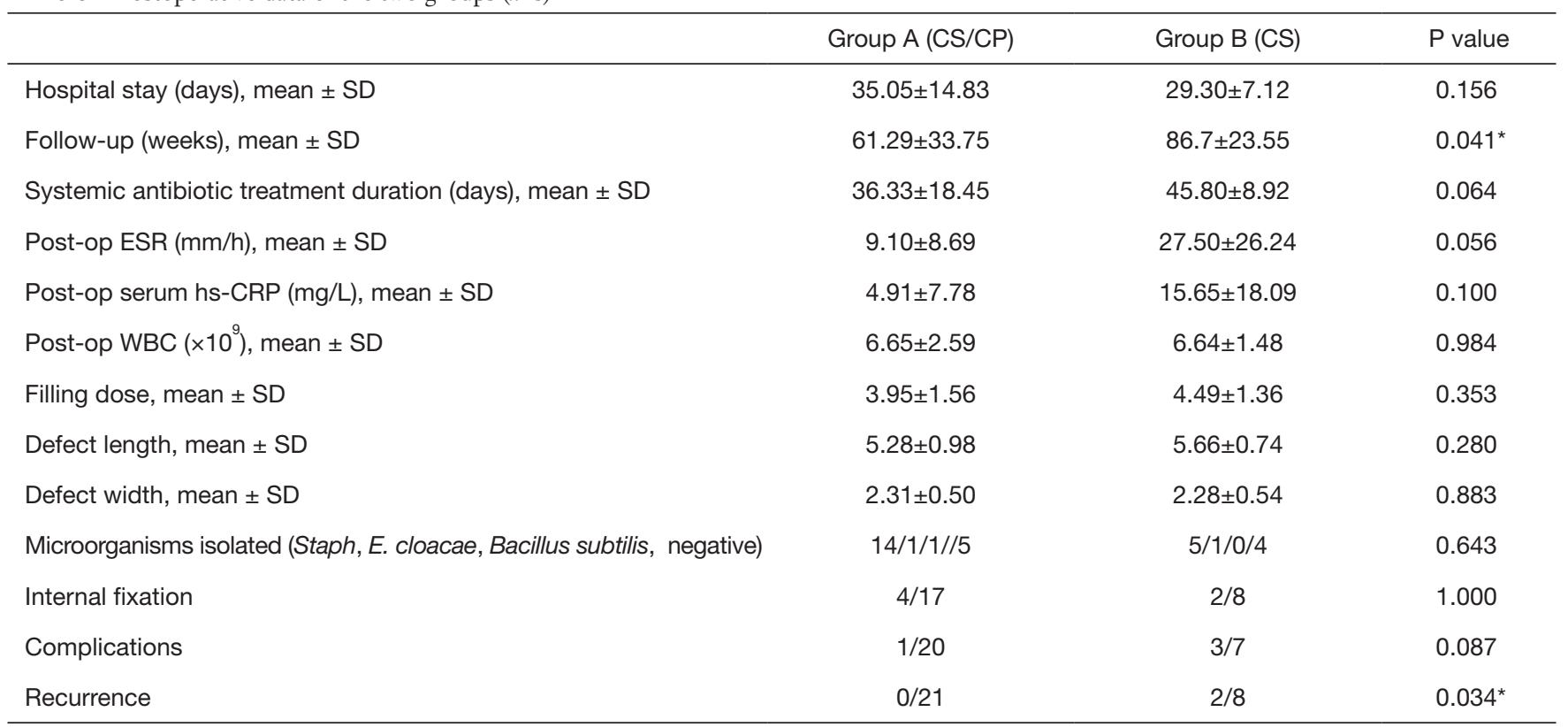

*, $\mathrm{P}<0.05$. ESR, erythrocyte sedimentation rate; hs-CRP, high-sensitivity C-reactive protein; WBC, white blood cell count; CS, calcium sulfate; CP, calcium phosphate.

with the width of $2.31 \pm 0.50 \mathrm{~cm}$. The mean stay in hospital was 35 days. Four patients received internal fixation to maintain limb stability. One case was treated with local flap transfer. In group B, the mean dose of CS and antibiotic load were $4.49 \mathrm{cc}$ mixed with vancomycin $(300 \mathrm{mg} / \mathrm{cc})$. The average length of defect was $5.66 \pm 0.74 \mathrm{~cm}$, with the width of $2.28 \pm 0.54 \mathrm{~cm}$. The mean stay in hospital was 29.3 days. Two patients received internal fixation (Table 2).

The microbial culture results indicated that staphylococcus aureus was the most frequently infected microorganism of all the patients. The mean duration of antibiotic use in group A was 36 days and 45 days in group B, and antibiotic sensitivity showed that all patients were sensitive to vancomycin. We analyzed the data collected and found no difference between the two groups except for distribution of bone defect $(\mathrm{P}=0.020)$ (Table 1).

\section{Anti-infection analysis}

In group $\mathrm{A}$, preoperative mean $\mathrm{ESR}, \mathrm{CRP}$, and $\mathrm{WBC}$ were $12.5 \mathrm{~mm} / \mathrm{h}, 15.3 \mathrm{mg} / \mathrm{L}$, and $6.3 \times 10^{9} / \mathrm{L}$, respectively, compared to that of $9.10 \mathrm{~mm} / \mathrm{h}, 4.91 \mathrm{mg} / \mathrm{L}$, and $6.65 \times 10^{9} / \mathrm{L}$ three months after operation. In group B, the mean ESR, CRP, and WBC were recorded to be $17.1 \mathrm{~mm} / \mathrm{h}, 5.8 \mathrm{mg} / \mathrm{L}$, and $6.85 \times 10^{9} / \mathrm{L}$ respectively, compared to that of $27.5 \mathrm{~mm} / \mathrm{h}, 15.65 \mathrm{mg} / \mathrm{L}$, and $6.64 \times 10^{9} / \mathrm{L}$ three months after operation. There were no statistical differences among all the indicators.

Recurrence and complication: no patient had recurrent infection in CS/CP group 17 months after surgery (Figure $2 A, B, C, D, E)$. While in group $\mathrm{B}, 2$ of the 10 cases during 18 months follow-up had infection recurrence ( 2 in tibia). They had incision crumbled and developed yellowish secretions after acquiring cold at week 5 after operation. Debridement drainage and antibiotics were used to treat the recurrence cases. One case (1 in calcaneum and forefoot) in CS/CP group and 3 cases ( 2 in femur and 1 in tibia) in CS group, who had delayed wound healing were cured after dress changing by using diluted iodophor saline. The infection of the rest in group B was effectively controlled in 1 year's follow-up. (Figure 2F,G,H,I,f). We found there was difference in the rates of recurrence between groups $(\mathrm{P}=0.034)$. However, complications like delayed wound healing in both groups showed no statistical differences $(\mathrm{P}=0.087)$.

\section{New bone formation and absorption analysis}

In group A $(\mathrm{CS} / \mathrm{CP})$ the average percentage of new bone formation was $20.5 \%, 43.7 \%$, and $75.2 \%$ at 1,3 , and 6 months after operation. In group B (CS), the average percentage of new bone formation was $15.4 \%, 32.2 \%$, and 

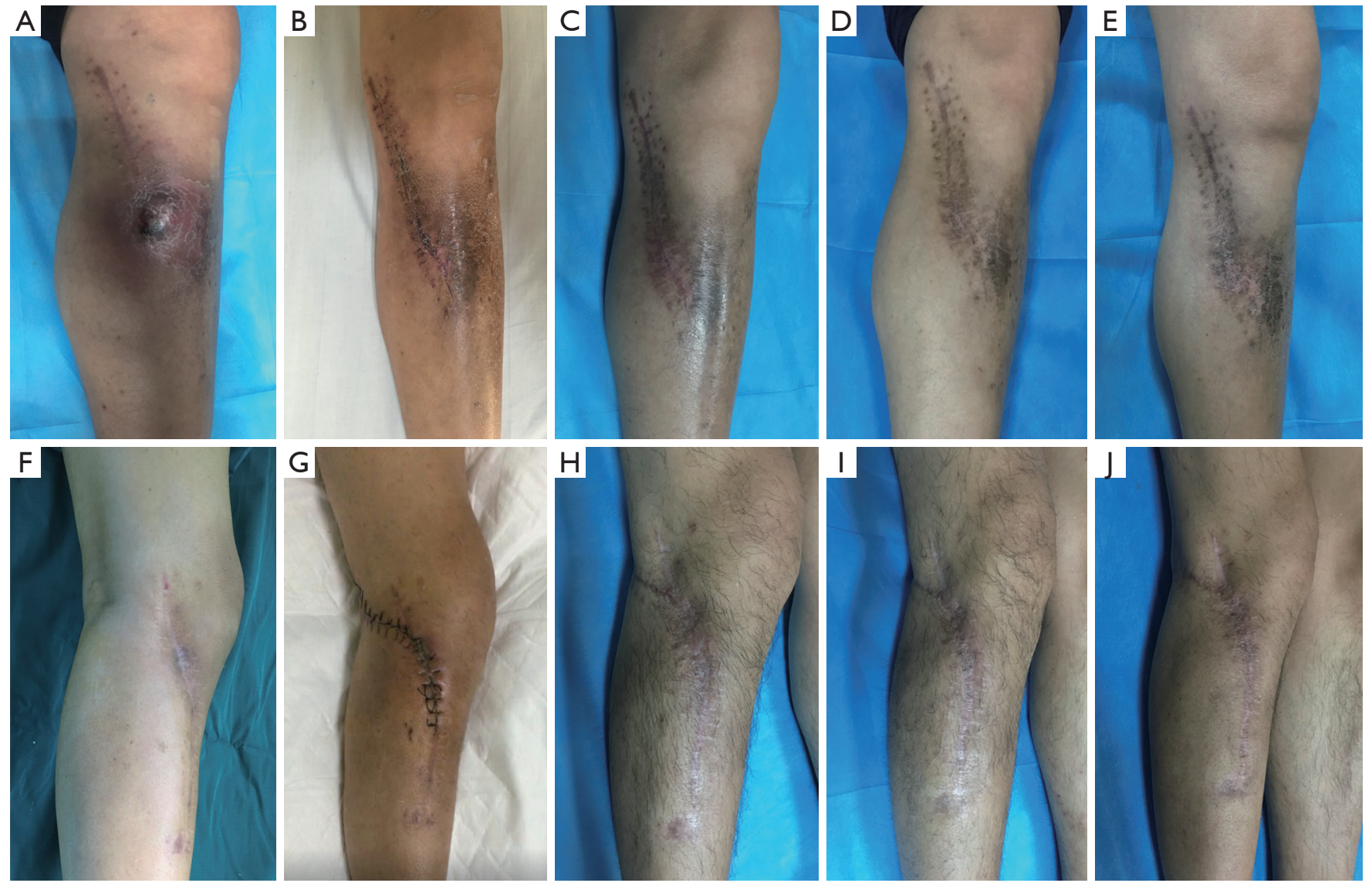

Figure 2 Wound healing in a patient with chronic osteomyelitis of the left proximal tibia treated with CS/CP composite preoperatively (A), immediately (B), at 3 months (C), at 6 months (D) and at 1 year (E) postoperatively. Wound healing in a patient with chronic osteomyelitis of the right proximal tibia was treated with CS preoperatively $(\mathrm{F})$, immediately $(\mathrm{G})$, at 3 months $(\mathrm{H})$, at 6 months $(\mathrm{I})$ and at 1 year $(\mathrm{J})$ postoperatively. CS, calcium sulfate; $\mathrm{CP}$, calcium phosphate.

Table 3 The average percentage of new bone formation and absorption $(\bar{x}+s)$

\begin{tabular}{lcc}
\hline Time & Group A (CS/CP) & Group B (CS) \\
\hline Bone formation of 1 month post-op & $0.205 \pm 0.021$ & $0.154 \pm 0.030$ \\
Bone formation of 3 months post-op & $0.437 \pm 0.093$ & $0.322 \pm 0.082$ \\
Bone formation of 6 months post-op & $0.752 \pm 0.094$ & $0.497 \pm 0.097$ \\
Bone absorption of 1 month post-op & $0.237 \pm 0.028$ & $0.471 \pm 0.056$ \\
Bone absorption of 3 months post-op & $0.564 \pm 0.080$ & $0.962 \pm 0.046$ \\
Bone absorption of 6 months post-op & $0.812 \pm 0.056$ & $1.000 \pm 0.000$ \\
\hline
\end{tabular}

${ }^{*}, \mathrm{P}<0.05$. CS, calcium sulfate; CP, calcium phosphate.

$49.7 \%$ at 1,3 , and 6 months after operation. Statistical results showed that there was significant difference in the average percentage of new bone formation after operation
$(\mathrm{P}=0.001, \mathrm{P}=0.025$, and $\mathrm{P}=0.000)$ (Table 3 and Figure 3). There were no significant correlations between substitute filling dose and new bone formation. Radiological results 

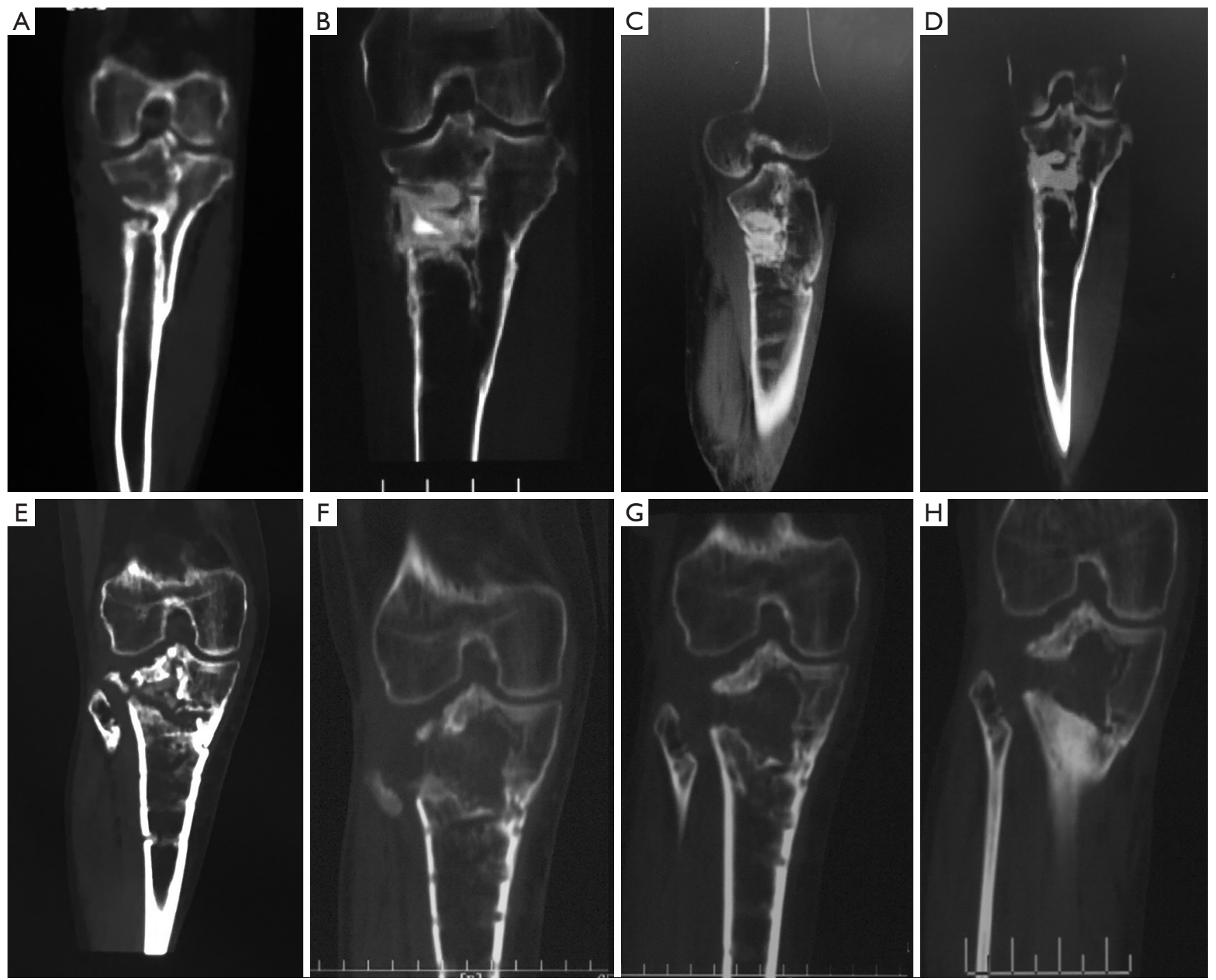

Figure 3 CT scans of chronic osteomyelitis treated with CS/CP obtained preoperatively (A), at 3 months (B), at 6 months (C) and at 1 year (D) postoperatively. It showed that the CS/CP composite was partially absorbed 3 months after surgery (B), and the rate of absorption was almost comparable to that of new bone formation. One year after surgery (D), the composite was completely absorbed and the new bone formation was almost complete, and the density of new bone was close to that of surrounding bone. CT scans of chronic osteomyelitis treated with CS were obtained preoperatively (E), at 3 months $(\mathrm{F})$, at 6 months $(\mathrm{G})$ and at 1 year $(\mathrm{H})$ postoperatively. It showed that the CS had been greatly dissolved at 3 months after surgery, and dissolved cavity was partly replaced by new bone, leaving new cavity (F). There was almost no apparent new bone formation and the defect still remained at 1 year's follow-up postoperatively $(\mathrm{H})$. CS, calcium sulfate; CP, calcium phosphate.

showed that the osteogenic ability of CS/CP group (Figure $3 A, B, C, D$ and Figure $4 A, B, C, D, E$ ) was better than that of CS group (Figure 3E,F, G,H and Figure 4F,G,H,I,f).

In group $\mathrm{A}$, the average percentage of bone substitute absorption was $23.7 \%, 56.4 \%$, and $81.2 \%$ at 1,3 , and 6 months after operation. In group B, the average percentage of composite absorption was $47.1 \%, 96.2 \%$, and $100 \%$ at 1 , 3 , and 6 months after operation. There was no significant correlation between substitute filling dose and bone absorption. Statistical results showed significant difference in the average percentage of composite absorption $(\mathrm{P}=0.000$, 0.000 , and 0.000) (Table 3). 

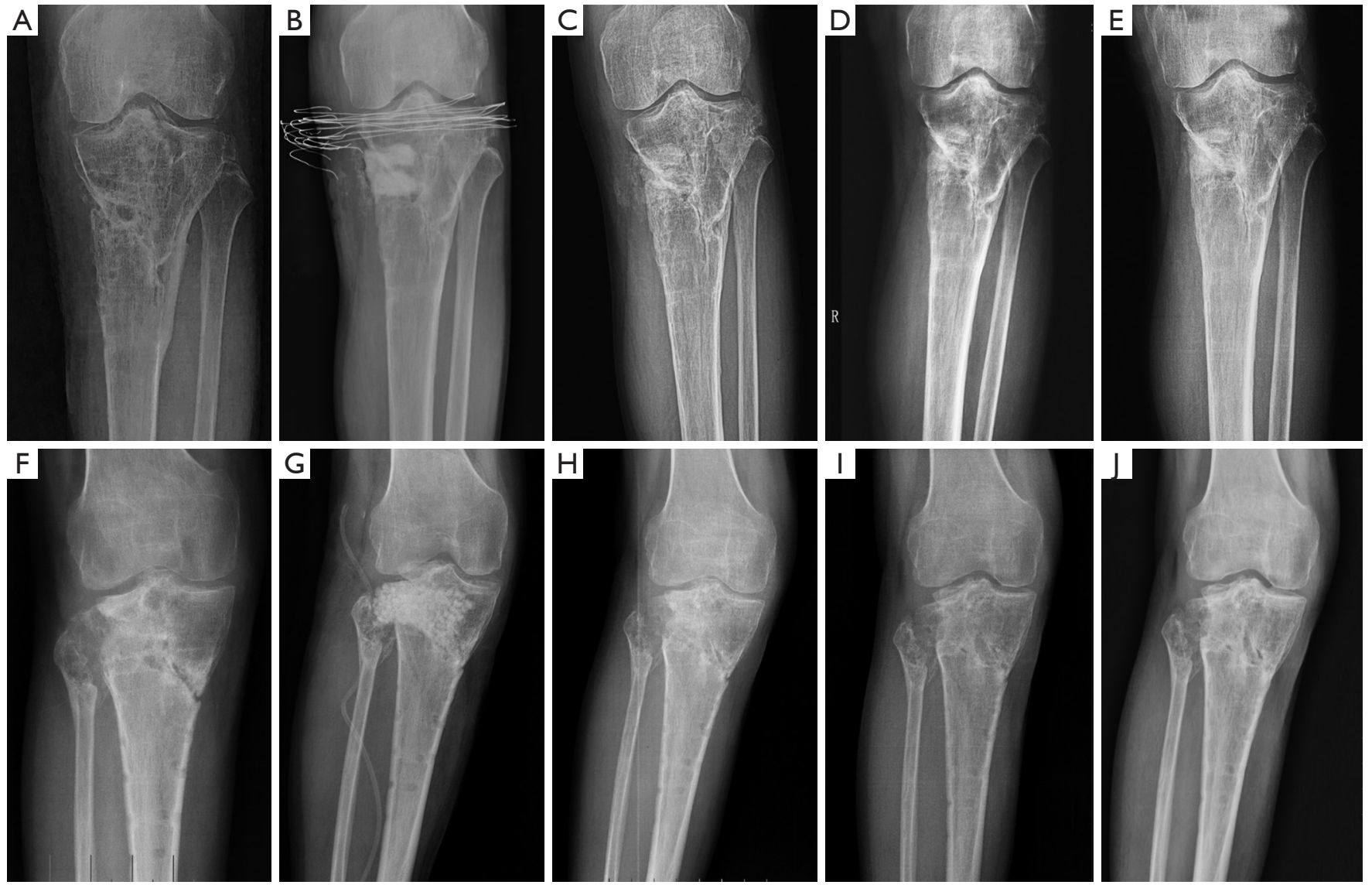

Figure 4 Typical radiographs of chronic osteomyelitis treated with CS/CP preoperatively (A), immediately (B), at 3 months (C), at 6 months (D) and at 1 year (E) postoperatively. With the formation of new bone, the bone substitute composite was gradually absorbed and no new defects formed until the formation of new bone completed (E). Typical radiographs of chronic osteomyelitis treated with CS preoperatively (F), immediately (G), at 3 months (H), at 6 months (I) and at 1 year (J) postoperatively. Three months after the surgery, CS had been greatly absorbed. However, the new bone formation was slow and bone defect cavities still existed $(\mathrm{H}, \mathrm{I}, \mathrm{J})$. CS, calcium sulfate; CP, calcium phosphate.

One case with calcaneal osteomyelitis treated by CS/CP composites, had a follow-up of 15 months postoperative. Imaging results of 1, 6, 12, 15 months after operation showed that as the substitute being absorbed, new bone gradually formed from periphery towards the center of defect which had almost similar density to surrounding normal bone (Figure 5). The latest X-ray and CT scan showed that more than $90 \%$ of the CS/CP composite was absorbed and new bone formed well.

\section{Discussion}

Infected bone defect can be induced by trauma, osteomyelitis or tumor and is still a great challenge. Principles to treat infected bone defect include: thorough debridement, antibiotics therapy and bone defect reconstruction. CS loaded with antibiotics has been successfully used for infected bone defects. However, bone formation and biomechanics property are not reliable and pathological fractures have been reported in some patients (3-5).

For the first time, bioabsorbable composite consists of CS and CP impregnated with vancomycin was introduced to treat chronic osteomyelitis. CS was used as comparation. Bone formation, bone absorption and anti-infection property were analyzed. 

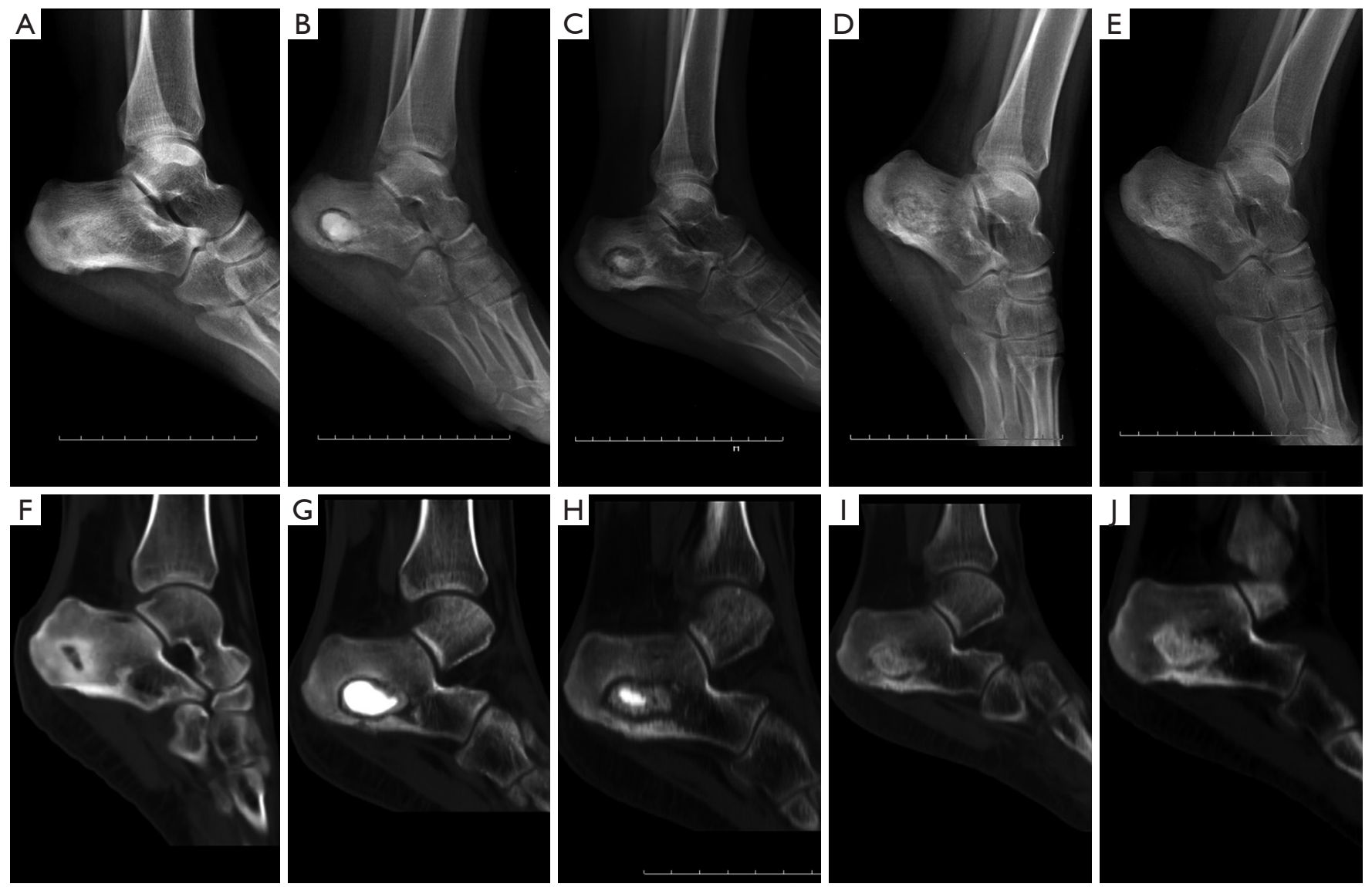

Figure 5 Female patient with calcaneal osteomyelitis of left foot. X Radiographs and CT scans of chronic osteomyelitis before operation (A,F). X Radiographs and CT scans of osteomyelitis treated with CS/CP obtained at 1 months (B,G), at 6 months $(\mathrm{C}, \mathrm{H})$, at 12 months $(\mathrm{D}, \mathrm{I})$ and at 15 months (E,J) postoperatively. It showed that at 15 months after surgery, the defect was almost fully filled with new bone, of which the density was almost equivalent to that of surrounding bone (E,J). CS, calcium sulfate; CP, calcium phosphate.

\section{New bone formation/absorption property}

The key indicator in our study was the rate of new bone formation, and this is the first time that we measured and calculated the rate of new bone formation in the treatment of chronic osteomyelitis by CS or CS/CP composite. We found that CS/CP composite has significantly higher percentage of new bone formation at 1 and 6 months compared with CS. Our results suggest that the osteogenic property of CS/CP was much more superior than CS alone.

In addition to the new bone formation rate, we calculated the average percentage of CS/CP absorption at 1,3 , 6 months after operation, and they were $23.7 \%, 56.4 \%$, $81.2 \%$, compared to that of CS with $47.1 \%, 96.2 \%, 100 \%$, which has statistical significance. The absorption rate of CS can reach almost $100 \%$ in 3 months post-operatively, meaning that CS absorbed too fast, and almost no apparent new bone formation occurred during absorption period, leading to new dead cavities created due to mismatch between absorption and osteogenesis. Meanwhile, the absorption rate of $\mathrm{CS} / \mathrm{CP}$ was almost the same as that of osteogenesis, leaving no new cavities. The new bone osteogenic capacity and substitute absorption rate of the two groups were similar with the results reported previously (19). Besides, CS/CP composite expected intermediate resorption kinetic and can be gradually replaced by host bone and new bone formed from periphery towards the center of defect (18). There was statistical difference in the site of bone defects between groups, however we do not regard it as influence on our outcomes, due to the reason that we paid more attention to the size of the defect, and 
selected cases of defect within a certain range according to inclusion and exclusion criteria to ensure the balance of our study. Meanwhile, there were no significant differences in other baseline data between groups (including etiology, age, preoperative and postoperative laboratory examination, hospital stay, size of defect, strategy of antibiotic treatment, bacterial distribution).

Therefore, The CS/CP composite graft can provide tailored resorption profile that would be slower resorbed than conventional CS alone but faster than CP alone of which residual graft can be seen for a long period or even permanently existed (32-36). This superior new bone formation efficiency of CS/CP was also confirmed by Nathan Evaniew, with rapid biological integration and no composite-related complications (26). Landgraeber applied $\mathrm{CS} / \mathrm{CP}$ in the treatment of avascular necrosis of the femoral head and found that it had properties of good osteogenesis, strong compression force and high stability (27).

\section{Anti-infection efficiency}

Both culture-specific systemic antibiotic and local antibiotic delivery are important treatment against chronic infection. Local delivery of antibiotics like tobramycin, vancomycin or gentamicin has been applied successfully in the treatment of chronic osteomyelitis $(2,37,38)$. Recently, biodegradable cements vectors like CS, CP or other material have shown improved elution of a wider range of antibiotics and are becoming more and more attractive when treating chronic osteomyelitis (39-41).

High local concentrations of antibiotics can not only offer significant bacteriocidal action against the planktonic microorganisms during the immediate calcaneus osteomyelitis debridement period, but also help to eradicate foci in macroscopically healthy bone which may harbor residual bacteria and reduce infection recurrence, besides it has been demonstrated that antibiotic-impregnated CS beads can prevent early bacterial colonization and biofilm formation by Methicillin-resistant Staphylococcus aureus in vitro, with long periods of sustained efficacy $(41,42)$.

Lindberg $F$ proved that the mixture of Cerament $G$ offers high local concentrations of gentamycin more than 100 times above the minimum inhibitory concentration (MIC) for gentamycin sensitive micro-organisms for the first 8 days and more than 10 times the MIC for the next 20 days (43). It has been proved that synthetic CS can release antibiotic for long-term, and address the function of biofilm prevention and bacterial eradication (41).
In our study, both CS and CS/CP showed anti-infection effect as antibiotic vectors. In CS group, 2 patients had infection relapses after acquiring cold, and then they received additional surgical treatment. We think that this may be partly attributed to shorter duration of antibiotic release due to enhanced CS absorption or incomplete debridement. Meanwhile, we found that there was no recurrence in CS/CP group, and this may be due to that the $\mathrm{CS} / \mathrm{CP}$ can release antibiotics continuously to keep a high local concentration with longer period. In addition, in CS/CP group, the new bone fully formed and bone defect was almost completely filled. Thus, CS/CP composite has better dead space management capacity which is helpful the control of infection.

Meanwhile, 3 patients developed delayed wound healing with wound leakage in group B, and were settled by continuous surgical dressing change. This may be related with fast resorption of CS. We do not use this leakage as an indication to re-operate and it is not directly related to recurrence of infection, which was agreeable with previous research $(37,44)$.

In this study, broad-spectrum vancomycin was used as the first choice over other antibiotics, as it has the least cytotoxic effect and the normal cell activity can be affected only when the local concentrations are too high (45). According to the results of bacterial culture and drug susceptibility, most cases in our study was sensitive to vancomycin. Which is similar to our research, the combination of CS and hydroxyapatite (HA) mixture loaded with antibiotics has also been successfully introduced as new bone substitute for calcaneal osteomyelitis (46).

ESR, CRP are usually used as indicators for infection commonly, however, our study showed no significant difference in ESR and CRP between two groups, and no significant difference in the same group pre/post operatively, suggesting that in the detection of inflammatory indicators of chronic osteomyelitis, the two indicators may not play a very significant role, which was similar to previous study (47). Apart from the indicators above, interlenkin-6 (IL-6), tumor necrosis factor (TNF- $\alpha$ ) and procalcitonin (PCT) also act as serum indicators for diagnosis of chronic osteomyelitis, and positive rates of diagnose were higher combined TNF- $\alpha$ with IL- 6 than WBC or CRP, yet so far have not been widely used in the diagnosis of chronic osteomyelitis (48). Besides, Güler showed in his study that PCT was more discriminative than other indicators, which was useful in the diagnosis of chronic osteomyelitis (49).

However, our study has some shortcomings. This is 
not a randomized control trial which has a relatively small number of cases in each group and the bone defect site is not concentrated. Hence, a larger-sample perspective study remained to be conducted in the future. Besides, antibiotics loading and elution kinetics require further research. Anyway, exploration of new bioactive bone substitutes combined with superior pharmacokinetics characteristics will be trend of future and represent a promising prospect.

\section{Conclusions}

In summary, for the first time, this novel CS/CP composition was analyzed in the treatment of chronic osteomyelitis. We proved that compared with CS, CS/CP acted as superior scaffold for bone formation with a lower rate of infection recurrence. This study provided evidence and basis for orthopedist and plastic surgeon when choosing $\mathrm{CS} / \mathrm{CP}$ or $\mathrm{CS}$ as bone substitutes in the therapy of chronic osteomyelitis.

\section{Acknowledgments}

Funding: This study was supported by Innovation Development Fund Project of Tangdu Hospital (TC201903 to Yunfei Zhang) and Military Medical Science and Technology Youth Training Program (18QNP022 to Yunfei Zhang).

\section{Footnote}

Conflicts of Interest: All authors have completed the ICMJE uniform disclosure form (available at http://dx.doi. org/10.21037/apm.2020.03.23). The authors have no conflicts of interest to declare.

Ethical Statement: The authors are accountable for all aspects of the work in ensuring that questions related to the accuracy or integrity of any part of the work are appropriately investigated and resolved. The study was conducted in accordance with the Declaration of Helsinki (as revised in 2013). The study was approved by institutional ethics board of Tangdu Hospital, Fourth Military Medical University (No. TDLL-201709-19). We obtained the informed consent of all subjects who were to participate in our study.

Open Access Statement: This is an Open Access article distributed in accordance with the Creative Commons
Attribution-NonCommercial-NoDerivs 4.0 International License (CC BY-NC-ND 4.0), which permits the noncommercial replication and distribution of the article with the strict proviso that no changes or edits are made and the original work is properly cited (including links to both the formal publication through the relevant DOI and the license). See: https://creativecommons.org/licenses/by-nc-nd/4.0/.

\section{References}

1. Beck-Broichsitter BE, Smeets R, Heiland M. Current concepts in pathogenesis of acute and chronic osteomyelitis. Curr Opin Infect Dis 2015;28:240-5.

2. Malkawi H, Shannak A, Sunna' P. Active treatment of segmental defects of long bones with established infection. A prospective study. Clin Orthop Relat Res 1984;184:241-8.

3. Haas DW, McAndrew MP. Bacterial osteomyelitis in adults: Evolving considerations in diagnosis and treatment. Am J Med 1996;101:550-61.

4. Kavanagh N, Ryan EJ, Widaa A, et al. Staphylococcal osteomyelitis: Disease progression, treatment challenges, and future directions. Clin Microbiol Rev 2018. doi: 10.1128/CMR.00084-17.

5. Kurien T, Pearson RG, Scammell BE. Bone graft substitutes currently available in orthopaedic practice. Bone Joint J 2013;95-B:583-97.

6. Offner D, Wagner Q, Keller L, et al. Complications d'une autogreffe osseuse, et comparaison avec une allogreffe osseuse ou l'utilisation de BMPs (Bone Morphogenetic Proteins): une revue systématique de la littérature. Le Journal de l'Orthopédie 2017;18:3032-43.

7. Fernandez de Grado G, Keller L, Idoux-Gillet Y, et al. Bone substitutes: a review of their characteristics, clinical use, and perspectives for large bone defects management. J Tissue Eng 2018;9:2041731418776819.

8. Fillingham Y, Jacobs J. Bone grafts and their substitutes. Bone Joint J 2016;98-B:6-9.

9. Yang D, Yan Y, Liu X, et al. Characterization of an $\alpha$-calcium sulphate hemihydrates/ $\alpha$-tricalcium phosphate combined injectable bone cement. ACS Appl Bio Mater 2018;1:768-76.

10. Roberts TT, Rosenbaum AJ. Bone grafts, bone substitutes and orthobiologics. Organogenesis 2012;8:114-24.

11. Kelly CM, Wilkins RM, Gitelis S, et al. The use of a surgical grade calcium sulfate as a bone graft substitute. Clin Orthop Relat Res 2001;382:42-50.

12. Cheung HS, Haak MH. Growth of osteoblasts on 
porous calcium phosphate ceramic: an in vitro model for biocompatibility study. Biomaterials 1989;10:63-7.

13. Frayssinet $\mathrm{P}$, Mathon $\mathrm{D}$, Lerch A, et al. Osseointegration of composite calcium phosphate bioceramics. J Biomed Mater Res 2000;50:125-30.

14. Gaasbeek RD, Toonen HG, van Heerwaarden RJ, et al. Mechanism of bone incorporation of $\beta$-TCP bone substitute in open wedge tibial osteotomy in patients. Biomaterials 2005;26:6713-9.

15. Bohner M. Calcium orthophosphates in medicine: from ceramics to calcium phosphate cements. Injury 2000;31:37-47.

16. Russell TA, Leighton RK. Comparison of autogenous bone graft and endothermic calcium phosphate cement for defect augmentation in tibial plateau fractures. A multicenter, prospective, randomized study. J Bone Joint Surg Am 2008;90:2057-61.

17. Xu R, Lian X, Li C, et al. Preparation and properties of calcium sulfate/tricalcium phosphate controllable bone cement. Journal of Functional Materials 2019;50:07105-10.

18. Civinini R, Capone A, Carulli C, et al. The kinetics of remodeling of a calcium sulfate/calcium phosphate bioceramic. J Mater Sci Mater Med 2017;28:137.

19. Urban RM, Turner TM, Hall DJ, et al. Increased bone formation using calcium sulfate-calcium phosphate composite graft. Clin Orthop Relat Res 2007;459:110-7.

20. Fillingham YA, Lenart BA, Gitelis S. Function after injection of benign bone lesions with a bioceramic. Clin Orthop Relat Res 2012;470:2014-20.

21. Zhu X, Chen X, Chen C, et al. Evaluation of calcium phosphate and calcium sulfate as injectable bone cements in sheep vertebrae. J Spinal Disord Tech 2012;25:333-7.

22. Guo H, Wei J, Liu CS. Development of a degradable cement of calcium phosphate and calcium sulfate composite for bone reconstruction. Biomed Mater 2006;1:193-7.

23. Turner TM, Urban RM, Gitelis S, et al. Radiographic and histologic assessment of calcium sulfate in experimental animal models and clinical use as a resorbable bonegraft substitute, a bone-graft expander, and a method for local antibiotic delivery: one institution's experience. J Bone Joint Surg Am 2001;83-A Suppl 2:8-18.

24. Hu G, Xiao L, Fu H, et al. Study on injectable and degradable cement of calcium sulphate and calcium phosphate for bone repair. J Mater Sci Mater Med 2010;21:627-34.

25. Hungerford DS. The use of an injectable calcium sulphate/calcium phosphate composite in the treatment of the osteonecrosis of the femoral head. J Bone J Surg Br 2009;91:331.

26. Evaniew N, Tan V, Parasu N, et al. Use of a calcium sulfate-calcium phosphate synthetic bone graft composite in the surgical management of primary bone tumors. Orthopedics 2013;36:e216-e222.

27. Landgraeber S, Theysohn JM, Classen T, et al. Advanced core decompression, a new treatment option of avascular necrosis of the femoral head--a first follow-up. J Tissue Eng Regen Med 2013;7:893-900.

28. Lazik A, Landgraeber S, Claßen T, et al. Aspects of postoperative magnetic resonance imaging of patients with avascular necrosis of the femoral head, treated by advanced core decompression. Skeletal Radiol 2015;44:1467-75.

29. Classen T, Warwas $S$, Jäger $M$, et al. Two-year follow up after advanced core decompression. J Tissue Eng Regen Med 2017;11:1308-14.

30. Mistry S, Roy S, Maitra, NJ, et al. A novel, multi-barrier, drug eluting calcium sulfate/biphasic calcium phosphate biodegradable composite bone cement for treatment of experimental MRSA osteomyelitis in rabbit model. J Control Release 2016;239:169-81.

31. Karr JC. An overview of the percutaneous antibiotic delivery technique for osteomyelitis treatment and a case study of calcaneal osteomyelitis. J Am Podiatr Med Assoc 2017;107:511.

32. Elsner A, Jubel A, Prokop A, et al. Augmentation of intraarticular calcaneal fractures with injectable calcium phosphate cement: densitometry, histology, and functional outcome of 18 patients. J Foot Ankle Surg 2005;44:390-5.

33. Keating JF, McQueen MM. Substitutes for autologous bone graft in orthopaedic trauma. J Bone Joint Surg Br 2001;83:3-8.

34. Ogose A, Hotta T, Kawashima H, et al. Comparison of hydroxyapatite and beta tricalcium phosphate as bone substitutes after excision of bone tumors. J Biomed Mater Res B Appl Biomater 2005;72:94-101.

35. Matsumine A, Kusuzaki K, Matsubara T, et al. Calcium phosphate cement in musculoskeletal tumor surgery. J Surg Oncol 2006;93:212-20.

36. Rougraff BT. Bone graft alternatives in the treatment of benign bone tumors. Instr Course Lect 2005;54:505-12.

37. Ferguson JY, Dudareva M, Riley ND, et al. The use of a biodegradable antibiotic-loaded calcium sulphate carrier containing tobramycin for the treatment of chronic osteomyelitis. Bone Joint J 2014;96-B:829-36.

38. Andreacchio A, Alberghina F, Paonessa M, et al. Tobramycin-impregnated calcium sulfate pellets for 
the treatment of chronic osteomyelitis in children and adolescents. J Pediatr Orthop B 2019;28:189-95.

39. Berebichez-Fridman R, Montero-Olvera P, Gómez-García $\mathrm{R}$, et al. An intramedullary nail coated with antibiotic and growth factor nanoparticles: An individualized stateof-the-art treatment for chronic osteomyelitis with bone defects. Med Hypotheses 2017;105:63-8.

40. Yan RJ, Zhang C, Guo QF, et al. One-stage compound grafting of antibiotic-impregnated calcium sulfate and autogenous cancellous bone for the treatment of chronic calcaneal osteomyelitis. Zhongguo Gu Shang 2014;27:854-7.

41. Howlin RP, Brayford MJ, Webb JS, et al. Antibiotic-loaded synthetic calcium sulfate beads for prevention of bacterial colonization and biofilm formation in periprosthetic infections. Antimicrob Agents Chemother 2015;59:111-20.

42. Attinger C, Wolcott R. Clinically addressing biofilm in chronic wounds. Adv Wound Care (New Rochelle) 2012;1:127-32.

43. Lindberg F, Lidén E, Sandell V. Antibiotic elution and bone remodelling with a novel bone substitute impregnated with Gentamicin. EBJIS Conference 2012.

Cite this article as: Zhao Z, Wang G, Zhang Y, Luo W, Liu S, Liu Y, Zhou Y, Zhang Y. The effect of calcium sulfate/ calcium phosphate composite for the treatment of chronic osteomyelitis compared with calcium sulfate. Ann Palliat Med 2020;9(4):1821-1833. doi: 10.21037/apm.2020.03.23
44. McNally M, Fergusson J, Kendall J, et al. A comparative study of three bioabsorbable antibiotic carriers in chronic osteomyelitis: 313 patients with minimum one-year followup. Bone Joint Proc 2015;97:21-2.

45. Rathbone CR, Cross JD, Brown KV, et al. Effect of various concentrations of antibiotics on osteogenic cell viability and activity. J Orthop Res 2011;29:1070-4.

46. Drampalos E, Mohammad HR, Kosmidis C, et al. Single stage treatment of diabetic calcaneal osteomyelitis with an absorbable gentamicin-loaded calcium sulphate/ hydroxyapatite biocomposite: The Silo technique. Foot (Edinb) 2018;34:40-4.

47. Leung AH, Hawthorn BR, Simpson AH. The effectiveness of local antibiotics in treating chronic osteomyelitis in a cohort of 50 patients with an average of 4 years follow-up. Open Orthop J 2015;9:372-8.

48. Jiang, N, Ma YF, Jiang Yi, et al. Clinical characteristics and treatment of extremity chronic osteomyelitis in southern china. Medicine 2015;94:e1874.

49. Güler EA, Kirmit A, Yıldırım S. Can procalcitonin help the diagnosis of osteomyelitis in adults? A prospective study. Ahi Evran Tip Dergisi 2017;1:25-9. 